\title{
Optimal Control of Beer Fermentation Process Using Differential Transform Method
}

\author{
*15HEHU, MD; ${ }^{2} \mathrm{JIYA}, \mathrm{M} ;{ }^{3}$ ELEBUTE, KO; ${ }^{4}$ AHMED, HO \\ ${ }^{1,2,3,4}$ Department of Mathematics, Federal University of Technology, Minna, Niger State, Nigeria \\ E-mail: m.shehu@futminna.edu.ng
}

\begin{abstract}
In this paper, the mathematical model of batch fermentation process of ethanol was formulated. The method of differential transform was used to obtain the solution governing the fermentation process; the system of equation was transformed using the differential transform method. The result obtained from the model was observed to show that temperature plays an important role in the fermentation of ethanol, with an increase in temperature the production of ethanol tends to decreases while with a low temperature the production of ethanol increases. @JASEM

https://dx.doi.org/10.4314/jasem.v21i4.16
\end{abstract}

Keyword: Optimal Ethanol, Fermentation, Substrate, Diacetyl, Inoculum

Fermentation was conventionally a process which enabled the preservation of food and as such has been used for centuries until present. Well nowadays, the main purpose of food fermentation is not to preserve, since other preservation techniques are known, but to produce a wide variety of fermentation products with specific taste, flavor, aroma and texture. (Chojnacka, 2006)

According to Claes and Van, (2014), fermentation is categorized into batch, fed- batch and continues fermentation which is based on the mode used in process operation. Fermentation processes are increasingly used in industries, laboratories and locally for the production of beverages, food, medicine, chemicals etc.

It is observed that an attempt to maximize the productivity whilst minimizing the production costs have gained interest by researchers in recent times. This present research is connected with the behavior of ethanol production based on temperature, $\mathrm{pH}$, dissolved oxygen and biological variables (biomass, substrate and product concentration) (Chojnacka, 2006).

In summary, we considered the variables that are most significant and in which way they affect the quality of the process or its products.

The proposed method of solution in this work is the differential transform method (DTM). The differential transform method can be classified as both an analytical and numerical method of solution for solving differential equations. The ideal of the differential transform was first proposed by Zhou,
(1986) and its main application is to solve both linear and nonlinear initial value problems. The DTM gives exact values of the $\mathrm{n}^{\text {th }}$ derivative of an analytical function at a point in terms of known and unknown boundary conditions. This method constructs, for differential equations, an analytical solution in the form of a polynomial (Kajani and Shehni, 2011).

The aim of this paper is to analyze the dynamics of batch fermentation process in the production of ethanol at different temperature using differential transform method as a method of solution.

Batch Fermentation Model: We are considering a kinetic model in this research work. The model uses the realistic aspects of the process such as the features of the wort and yeast, and also two important byproducts of fermentation: ethyl acetate and diacetyl.

The model takes into account five responses: biomass $(X)$, substrate ( $S$ ), ethanol ( $e$ ), diacetyl(diac) and ethyl acetate(ecet).

The biomass is divided into three different types of cells and they include latent $\left(x_{\text {lag }}\right)$, active $\left(x_{\text {active }}\right)$ and dead $\left(x_{\text {dead }}\right)$ cells. The process of fermentation was divided into two phases, that is, the lag phase and the fermentation phase. At the initial state, when the activation processes start, no fermentation is observed. So, it is easy to explain the lag phase as a phase in which only two processes can be observed: dead cells are setting down to the bottom and lag cells are being activated. 


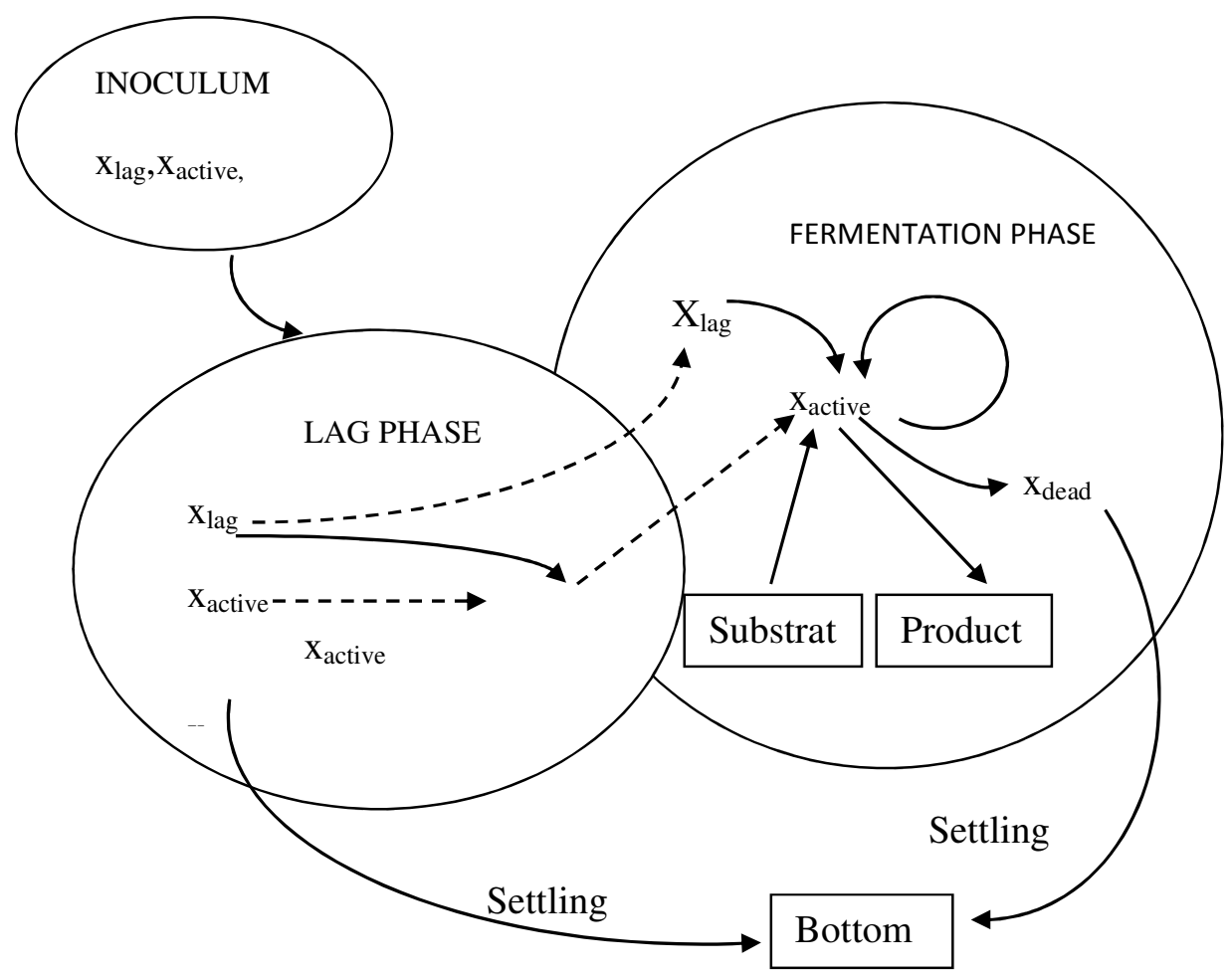

Fig 1: The Model Diagram for the Fermentation Process

According to de Andres Toro et al. (2014), fermentation and growth start when about $80 \%$ of lag cells have been transformed into active cells, which begin the fermentation phase. The time at which the lag phase ends and the fermentation phase begin was name $t_{\text {lag }}$

The bi-products with an important impact in the beer fermentation are: ethyl acetate which gives the beer a fruity odour and flavour, and diacetyl that make the beer heavy and also gives a sweet taste. It is assumed that the inoculum $\left(X_{\text {inc }}\right)$ is composed of about $50 \%$ of dead cells, $48 \%$ of lag cells and $2 \%$ of active cells.

From the fermentation model in figure 1, the lag and fermentation phase is given by the following equations:

$$
\begin{aligned}
& \frac{d x_{\text {lag }}}{d t}=-\gamma \cdot x_{\text {lag }} \\
& \frac{d x_{\text {active }}}{d t}=\varphi \cdot x_{\text {active }}-k_{m} \cdot x_{\text {active }}+\gamma \cdot x_{\text {lag }} \\
& \frac{d x_{\text {dead }}}{d t}=k_{m} x_{\text {active }}-\alpha x_{\text {dead }} \\
& \frac{d s}{d t}=-\phi x_{\text {active }} \\
& \frac{d e}{d t}=-\mu f x_{\text {active }}
\end{aligned}
$$


Table 1: Description of State Parameter Used in the Model

\begin{tabular}{cll}
\hline Parameters & Description & Unit \\
\hline$\mu$ & Ethanol production rate & $h^{-1}$ \\
$\gamma$ & Specific rate of latent fermentation & $h^{-1}$ \\
$\boldsymbol{\alpha}$ & Specific yeast settling down rate & $g / l$ \\
$\boldsymbol{\phi}$ & Ethyl acetate coefficient rate & $h^{-1}$ \\
$\boldsymbol{\varphi}$ & Substrate consumption rate & $h^{-1}$ \\
Acet & Specific yeast growth rate & $h^{-1}$ \\
Diac & Ethyl acetate concentration & $\mathrm{Ppm}$ \\
$f$ & Diacetyl concentration & $\mathrm{Ppm}$ \\
$k_{m}$ & Fermentation inhibitor factor & $g / l$ \\
$k_{s}$ & Yeast growth inhibitor parameter & $g / l$ \\
$s_{0}$ & Sugar inhibitor parameter & $g / l$ \\
$t$ & Initial concentration of sugar & $g / l$ \\
$T$ & & \\
\hline
\end{tabular}

Table 2: Description of the State Variable

\begin{tabular}{ll}
\hline Variables & Description \\
\hline$x_{\text {lag }}$ & Suspended latent biomass \\
$x_{\text {dead }}$ & Suspended dead biomass \\
$x_{\text {active }}$ & Suspended active biomass \\
$\boldsymbol{S}$ & \\
$\boldsymbol{e}$ & Concentration of sugar \\
$X$ & Ethanol concentration \\
\hline
\end{tabular}

The parameters used in equation (1) - (7) are calculated using the following:

$X=x_{\text {lag }}+x_{\text {active }}+x_{\text {dead }}$

$\varphi=\frac{\varphi_{0} s}{0.5\left(s_{0}\right)+e}$

$\alpha=\frac{0.5\left(s_{0}\right)+\alpha_{0}}{0.5\left(s_{0}\right)+e}$

$\phi=\frac{\phi_{0} s}{k_{s}+s}$ 


$$
\begin{aligned}
& \mu=\frac{\mu_{0} s}{k_{s}+s} \\
& f=1-\frac{e}{0.5 s_{0}}
\end{aligned}
$$

Since ethanol fermentation depend on temperature, the value of parameters in equation $7-12$ are calculated using the exponential equation of the Arrhenius type

$$
\omega=A \cdot \ell^{-\frac{E}{R T}}
$$

The constant value of $k_{d c}$ and $k_{d m}$ were calculated using the experimental data of diacetyl concentration behaviour. (Oonsivilai and Oonsivilai, 2010)

Application of Differential Transform Method: The differential transform method (DTM) used in this work is defined as follow;

$F(k)=\frac{1}{k !}\left[\frac{d^{k} f(x)}{d x^{k}}\right]_{x=x_{0}}$

Where, $f(\mathrm{x})$ is the original function and $F(\mathrm{k})$ is the transformed function.

The differential inverse transform of $F(\mathrm{k})$ is defined as;

$$
f(x)=\sum_{k=0}^{\infty} F(k)\left(x-x_{0}\right)^{k}
$$

From equations 14 and 15, we get

$$
f(x)=\left.\sum_{k=0}^{\infty} \frac{\left(x-x_{0}\right)^{k}}{k !} \frac{d^{k} f(x)}{d x^{k}}\right|_{x=x_{0}}
$$

Let

$$
x_{\text {lag }}=y_{1,} x_{\text {active }}=y_{2}, x_{\text {dead }}=y_{3}, s=y_{4 \text { and }} e=y_{5}
$$

Then, equation (1) - (5) becomes;

$$
\begin{gathered}
\frac{d y_{1}}{d t}=-\gamma y_{1} \\
\frac{d y_{2}}{d t}=\varphi y_{2}-k_{m} y_{2}+\gamma y_{1} \\
\frac{d y_{3}}{d t}=k_{m} y_{2}-\alpha y_{3} \\
\frac{d y_{4}}{d t}=-\varphi y_{2}
\end{gathered}
$$


$\frac{d y_{5}}{d t}=\mu f y_{2}$

Evaluating equation (17) - (21) at different sampled temperature considering low and high temperature profile, we obtain table (3)

Table 3: Values of Parameter Evaluated at Different Temperature.

\begin{tabular}{cccc}
\hline & Low Temperature & \multicolumn{2}{c}{ High Temperature } \\
\hline Parameter & $10^{0} C$ & $35^{0} C$ & $45^{0} C$ \\
$\varphi_{0}$ & 0.0114 & 107.8372 & 2801.5977 \\
$k_{m}$ & 0.0058 & 338.7897 & 16872.6767 \\
$\delta$ & 0.0185 & 37.8647 & 570.3238 \\
$\alpha_{0}$ & 0.3688 & 3.5376 & 9.8441 \\
$\phi_{0}$ & 0.4678 & 0.0166 & 0.005 \\
$\mu_{0}$ & 0.3004 & 0.4319 & 0.4915 \\
$\gamma$ & 0.0588 & 0.8956 & 2.3607 \\
$k_{s}$ & 3.2217 & $1.7794 \times 10^{-5}$ & $5.4337 \times 10^{-6}$
\end{tabular}

Now, taking the differential transform of equation (1) - (5), we have;

$$
\begin{aligned}
& \mathrm{Y}_{1}(\mathrm{k}+1)=\frac{1}{\mathrm{k}+1}\left[-0.0588 Y_{1}(\mathrm{k})\right] \\
& \mathrm{Y}_{2}(\mathrm{k}+1)=\frac{1}{\mathrm{k}+1}\left[0.0588 Y_{1}(\mathrm{k})-0.0058 \mathrm{Y}_{2}(\mathrm{k})+2.2344 \times 10^{-4} \mathrm{Y}_{3}(\mathrm{k})-4.3829 \times 10^{-6} \sum_{l=0}^{k} Y_{5}(l) Y_{3}(k-l)\right] \\
& \mathrm{Y}_{3}(\mathrm{k}+1)=\frac{1}{\mathrm{k}+1}\left[0.0058 \mathrm{Y}_{2}(\mathrm{k})-0.3687 \mathrm{Y}_{3}(\mathrm{k})+7.2314 \times 10^{-3} \sum_{l=0}^{k} Y_{5}(l) Y_{3}(k-l)\right] \\
& \mathrm{Y}_{4}(\mathrm{k}+1)=\frac{1}{\mathrm{k}+1}\left[-0.1452 \sum_{l=0}^{k} Y_{4}(l) Y_{2}(k-l)+0.0451 \sum_{m=0}^{k} \sum_{l=0}^{m} Y_{4}(l) Y_{4}(m-l) Y_{2}(k-m)\right] \\
& Y_{5}(k+1)=\frac{1}{k+1}\left[0.0932 \sum_{l=0}^{k} Y_{2}(\mathrm{l}) Y_{4}(k-l)-1.8275 \times 10^{-3} \sum_{m=0}^{k} \sum_{l=0}^{m} Y_{2}(1) Y_{4}(\mathrm{~m}-l) \mathrm{Y}_{5}(k-m)\right. \\
& \left.-0.0289 \sum_{m=0}^{k} \sum_{l=0}^{m} Y_{2}(1) Y_{4}(\mathrm{~m}-l) \mathrm{Y}_{4}(k-m)+5.4898 \times 10^{-4} \sum_{m=0}^{k} \sum_{n=0}^{m} \sum_{l=0}^{n} Y_{2}(\mathrm{l}) Y_{4}(\mathrm{n}-l) \mathrm{Y}_{4}(\mathrm{~m}-\mathrm{n}) \mathrm{Y}_{5}(k-m)\right]
\end{aligned}
$$

Evaluating equation (22) - (26) at different iteration of $k$, we have the table below; 


\begin{tabular}{cccccc}
\hline \multicolumn{6}{l}{ Table 4: Transform of $\mathrm{Y}(\mathrm{k}+1)$ at Different Value of $k$} \\
\hline $\mathrm{k}$ & $Y_{1}(\mathrm{k}+1)$ & $Y_{2}(\mathrm{k}+1)$ & $Y_{3}(\mathrm{k}+1)$ & $Y_{4}(\mathrm{k}+1)$ & $Y_{5}(\mathrm{k}+1)$ \\
0 & -0.1176 & 0.1153 & -0.9955 & -371.657 & 238.147 \\
1 & 0.0035 & 0.003 & -1.2907 & -1118.8734 & 4497.1851 \\
2 & -0.00007 & 0.017 & -27.8846 & -3362.5187 & 20836.454 \\
3 & $9.9616 \times 10^{-7}$ & 0.0477 & -78.7833 & -10109.129 & 97241.3965 \\
4 & $-1.1715 \times 10^{-8}$ & 0.2799 & 461.792 & -30398.55 & $2.6699 \times 10^{5}$ \\
5 & $1.14805 \times 10^{-10}$ & 0.4851 & 799.958 & -32769.6195 & $3.4219 \times 10^{5}$ \\
6 & $-9.6436 \times 10^{-13}$ & 0.7076 & 1166.7421 & -15189.7885 & $-1.7620 \times 10^{5}$ \\
\hline
\end{tabular}

The above table was obtained by evaluating equation (22) - (26) at various value of $k$ ranging from (0-6). Therefore, the closed form of solution to the differential transform in equation (22) - (26) using the values of $Y_{i}(\mathrm{k}+1)$ obtain in the above table, can be written as;

$$
\begin{aligned}
y(\mathrm{t}) & =\sum_{k=0}^{\infty} Y(k) t^{k} \\
& =Y(0)+\mathrm{tY}(1)+\mathrm{t}^{2} Y(2)+\mathrm{t}^{3} Y(3)+\mathrm{t}^{4} Y(4)+\ldots
\end{aligned}
$$

Hence;

$$
\begin{array}{ll}
y_{1}(\mathrm{t})=-0.1176+0.0035 t-0.00007 t^{2}+9.9616 \times 10^{-7} t^{3}-1.1715 \times 10^{8} t^{4}+1.1481 \times 10^{-10} t^{5}-9.6436 \times 10^{-13} t^{6} & 29 \\
y_{2}(\mathrm{t})=0.1153-0.003 t+0.017 t^{2}+0.0477 t^{3}+0.2799 t^{4}+0.4851 t^{5}+0.7076 t^{6} & 30 \\
y_{3}(\mathrm{t})=-0.9955-1.2907 t-27.8846 t^{2}-78.7833 t^{3}+461.792 t^{4}+799.958 t^{5}+1166.7421 t^{6} & 31 \\
y_{4}(\mathrm{t})=-371.657-1118.8734 t-33625187 t^{2}-10109.129 t^{3}-30398.55 t^{4}-32769.6195 t^{5}-15189.7885 t^{6} & 32 \\
y_{5}(\mathrm{t})=238.147+4497.1851 t+20836.454 t^{2}+97241.3965 t^{3}+26699 \times 10^{5} t^{4}+3.4219 \times 10^{5} t^{5}-1.762 \times 10^{5} t^{6} & 33
\end{array}
$$

Table 3.4 gives the numerical results (29) - (33) for our model equation (1) - (5) at a temperature of $10^{0} \mathrm{C}$.

\section{RESULT AND DISCUSSION}

Using the above method of solution at different temperature $\left(35^{\circ} C, 45^{\circ} C\right)$ we obtain the following figures below.

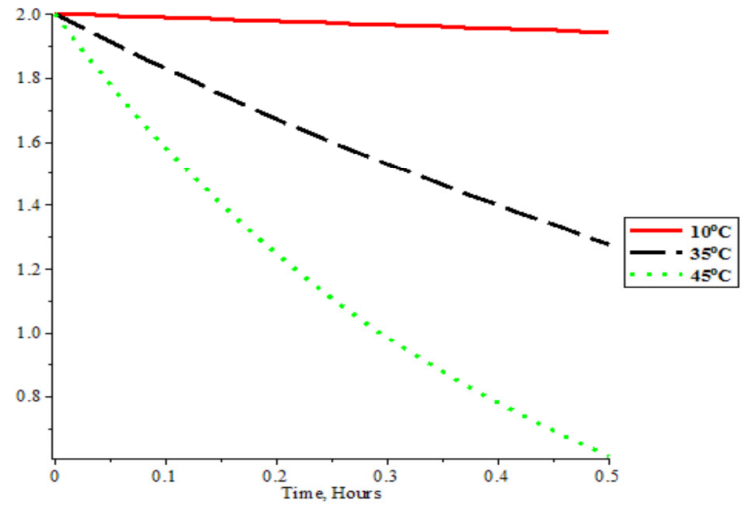

Fig 2: Graph of Lag Biomass $\left(\mathrm{x}_{\mathrm{lag}}\right)$ at Different Temperature 
Figure 2 shows the concentration of active biomass against time in hours at different temperature using equation (30). This graph shows that at the lag phase the active biomass concentration decreases with high change in temperature faster.

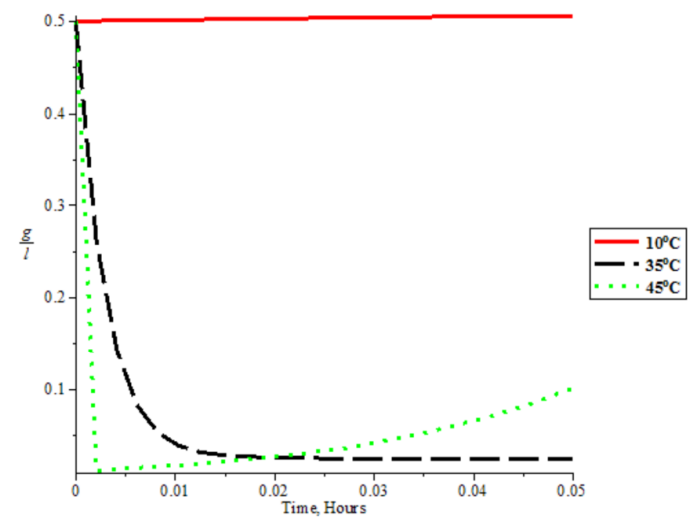

Fig 3: Graph of Active Biomass ( $\mathrm{x}_{\text {Active }}$ ) at Different Temperation

Figure 3 shows the concentration of dead biomass against time in hours at different temperature using equation (31). This graph shows that as the fermentation progresses the dead biomass concentration increases with time at high temperature while at low temperature the reduction seems to be insignificant.

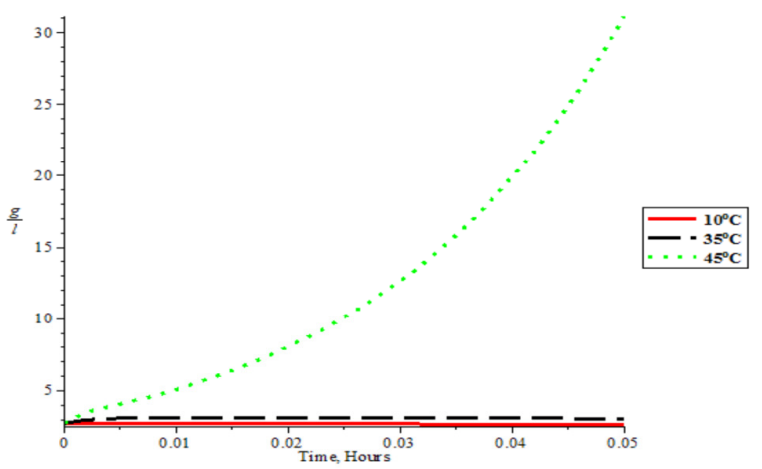

Fig 4: tGraph of Ethanol production against time in hours at different Temperature

Figure 4 shows the graph of ethanol production against time in hours at different Temperature using equation (32), it can be seen from the graph that as the temperature increases there is no significant change in the production of ethanol.



Fig 5: The Graph of Ethanol Production Against Time at Different Temperature

Figure 5 shows the concentration of sugar against time in hours at different temperature using equation (33). This graph shows that as the fermentation progresses at a low temperature the sugar concentration reduces faster with time but at high temperature there was no reduction in sugar which implies the yeast that convert sugar into ethanol tends to be in effective. 


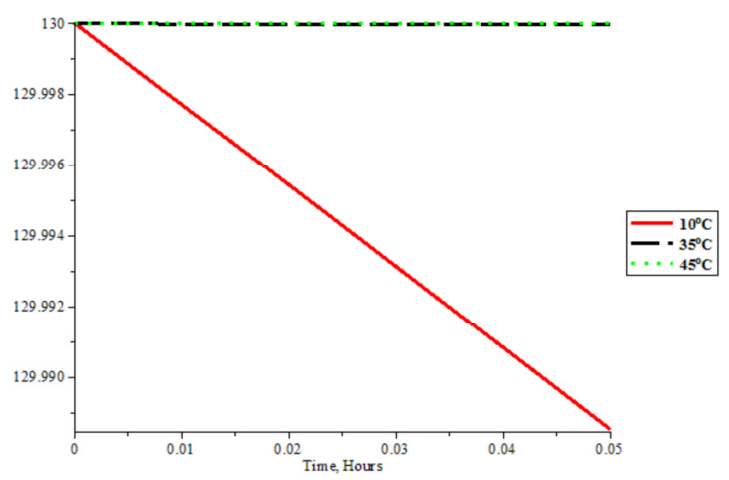

Fig 6: The Graph of Sugar Concentration Against Time At Different Temparature

Conclusion: We analysed the fermentation model of beer using differential transform technique as a method of solution, the result obtained shows that the differential transform method can be used to solve these type of system of differential equation efficiently. From the analysis we were able to see that temperature plays an important role in the production of ethanol, the lower the temperature the higher is the ethanol produce and vice versa.

\section{REFERENCES}

Chojnacka, K. (2006). Fermentation products, chemical engineering and chemical process technology. Wroclaw, Poland. Wroclaw University of Technology.

Claes, J. E. and Van Impe, J. F. (1999). On-line estimation of the specific growth rate based on viable biomass measurements experimental validation. Bioprocess Engineering, 21: 389395 doi 10.1007/s004490050692

De. Andres- Toro, B., Giron- Sierra, J. M., LopezOrozco, J. A., Fernandez- Conde, C.,
Peinado, J. M., and Garcia- Ochoa, F. (2014). Elsevier Journal of mathematics and computer in simulation. 48(2015) 65 - 74.

Kajani, M. T. and Shehni, N. A, (2011). Differential transform method: an effective tool for solving nonlinear volterra integro - differential equations, Australian Journal of Basic and Applied Sciences. 5: 30-39

Oonsivilai, R.and Oosnivilai, A. (2010). Temperature profile in fermentation process using differential evolution. Recent advances in energy and environment. ISSN: 1790-5095, pp 315 -319 .

Zhou, J. K. (1986). Differential transform and application for electrical circuits, Hoazhong University press, Wuhan, China. 